\title{
Prophylactic Bactericidal Orthopedic Implants - Animal Testing Study
}

\author{
Richard A. Wysk ${ }^{1}$, Wayne J. Sebastianelli ${ }^{1}$, Rohan A. Shirwaiker ${ }^{2}$, Gregory M. Bailey ${ }^{1}$, Charumani \\ Charumani $^{2}$, Mary Kennett ${ }^{2}$, Amy Kaucher ${ }^{2}$, Rachel Abrahams ${ }^{2}$, Thomas A. Fuller ${ }^{1 *}$, Patricia Royer, \\ Robert C. Voigt ${ }^{1}$ and Paul H. Cohen ${ }^{1}$
}

\footnotetext{
${ }^{1}$ ArgentumCidalElectrics (ACE), Inc., Lewistown, USA;

${ }^{2}$ The Pennsylvania State University, University Park, USA.

Email: *tafuller125@,hotmail.com; *fuller@argentumce.com
}

Received 3 January 2010; revised 18 February 2010; accepted 22 April 2010.

\begin{abstract}
This paper summarizes preliminary rat studies aimed at identifying the effectiveness of using electrically stimulated silver as a bactericidal agent for indwelling residual hardware devices (RHD). A variety of bactericidal indwelling devices were designed, fabricated and surgically inserted into the medullary cavity of live rats. The rats were inoculated with Staphylococcus aureus to try and induce osteomyelitis. A total of 37 surgeries were performed by implanting the rats with both control and potentially bactericidal devices. As surgical procedures and devices were improved, it appeared that the implants produced antibiotic effects in the animals. All of the control animals and all of the animals where the device failed tested positive for Staphylococcus aureus growth. Of the rats with operational bactericidal devices (those that survived the surgery and incubation period), half tested negative for Staphylococcus aureus. The device designs are discussed in this paper along with the test procedures, operating practices and results. A statistical analysis of the results, which shows a very high confidence level in the effectiveness of electrically stimulated silver as a bactericidal agent/antibiotic, is also presented.
\end{abstract}

Keywords: Antibacterial; Antimicrobial; Bactericidal; Silver; Ionic Silver; Residual Hardware Devices (RHD); Animal Testing

\section{INTRODUCTION}

Replacing arthritic joints has improved the quality of life for millions of Americans. Over the past decade, there has been an increase in the number of total hip and knee replacement surgeries performed in the U.S. In 2005, a total of 808,000 total hip and knee replacements were performed in the U.S [1]. By 2030, the total number of replacements is projected to be more than 4 million [2]. Of these surgeries, $0.3-2 \%$ result in deep bone infections (osteomyelitis), according to current data [3,4]. While this rate is fairly low, the cost associated with mitigating deep bone infections far exceeds the cost of the initial replacement surgery.

Patients who develop osteomyelitis must undergo difficult and expensive treatment. Total mitigation of osteomyelitic infection is typically achieved after: 1) implant device removal, 2) local debridement of infected area, 3) insertion of a spacer prosthetic, 4) an aggressive six week course of limb protection and aggressive antimicrobial therapy, and 5) a second joint arthroplasty. The primary objective of this research is to improve the quality of life by reducing pathogenic bacteria in in vivo joint replacements using the antimicrobial properties of silver stimulated by an electric current. The long-term goal is to augment current implants with antimicrobial surface technology, thus allowing treatment of RHD associated osteomyelitic infections without the removal of prosthetic implant or hardware device.

Significant research has been performed recently on antimicrobial colloids composed of silver nano-particles stabilized by polymers or other agents [5-7]. Many studies have shown these materials to display antimicrobial efficacy to a wide spectrum of microorganisms [8-10]. Our in vitro laboratory studies have shown these new materials to exhibit acceptable early antibacterial properties; however in water-rich environs, these materials may quickly lose their effectiveness (typically minutes to hours). After years of investigating the bactericidal effects of silver, the proper device configuration required for silver to be an effective bactericide has been identified. The key is to continually produce a controlled release of silver ions $(\mathrm{Ag}+)$ and expose bacteria to these ions for extended time periods.

Forbes [11] reported that $\$ 30$ billion is spent annually 
to fight infections, and that drug resistant infections kill more Americans every year than AIDS and breast cancer combined. Our hypothesis is that ionized antimicrobial silver and silver forms can be used to kill a wide variety of bacteria, i.e., both standard strains of bacteria as well as methicillin-resistant bacteria. We base this hypothesis on the long history of bactericidal metals, particularly silver, gold and copper, that have been used in medicine for years as antimicrobial agents in the form of wound dressings and debriding agents [8,9,12-14]. The historical problem has been that these bactericidal metals seem to work in certain situations while showing little effect in others. Based on three plus years of in vitro testing, we have identified the key science and engineering elements that allow bactericidal metals, particularly silver, to work effectively. The use of antimicrobial silver is particularly promising because in spite of being used for more than 100 years, there has been no evidence of a definitive pattern for silver resistant infections [15].

The principal focus of this research is to describe a new system designed to make replacement prostheses antibacterial. In the following section, we will describe the proposed system that is identified as effective against most bacteria which cause osteomyelitic infections. A few device design iterations along with a final device design that was used in our animal testing are also described. Finally, the protocol used for animal testing and the results that were obtained in the animal tests are presented.

\section{THE ANTIMICROBIAL SYSTEM}

A system was developed to provide prophylactic and antibiotic protection for both soft tissues as well as bone against common bacteria, e.g., strains of Escherichia coli, Staphylococcus aureus, Pseudomonas aeruginosa, Enterococcus faecalis, and methicillin-resistant Staphylococcus aureus (MRSA) and fungi e.g. Candida albicans. Our in vitro and early animal testing has shown that a properly configured device that is stimulated by a very small amount of electrical current is both an effective bactericide as well as a fungicide. The central hypothesis behind the proposed research is that electrically ionized antimicrobial silver and its alloys can be used as a safe and effective means of eliminating microorganisms associated with biofilms on RHDs. The key to this technology is using the bacteria-rich environment to carry silver ions and complete an electrical circuit in the device configuration shown in Figure 1. This configuration creates a regional antibiotic environment around the RHD (inhibition zone diameter $\approx 20 \mathrm{~mm}$ in a petri dish), and appears to work for different concentrations of bacterial colonies.

\section{IN VITRO STUDIES}

For more than three years, we have experimented with si lver stimulated by small currents to produce silver ions. We have demonstrated that our configuration is effective against all common harmful bacteria and fungi that were tested in the laboratory. Our system shows reproducible results for a variety of bacteria and fungi, as shown in Figure 2. In another test, we inserted our device in the medulary cavity of a rat tibia and then embedded the tibia in Mueller Hinton agar (MHA) inoculated with Pseudomonas aeruginosa to determine if our system worked in this simulated in vivo environment. The results of this test are shown in Figure 3. These results are significant in that the bactericidal kill region that is created from within the rat tibia extends well into the agar. This demonstrates that our $\mathrm{Ag}^{+}$system can penetrate the bone - a problem for most antibiotics.

\section{IN VIVO STUDIES-ANIMAL TESTING}

The properties of silver have been known for quite some time. Chronic exposure to silver, termed argyria, is manifested by an irreversible gray or blue-gray discoloration of the skin and mucous membranes. When the body is

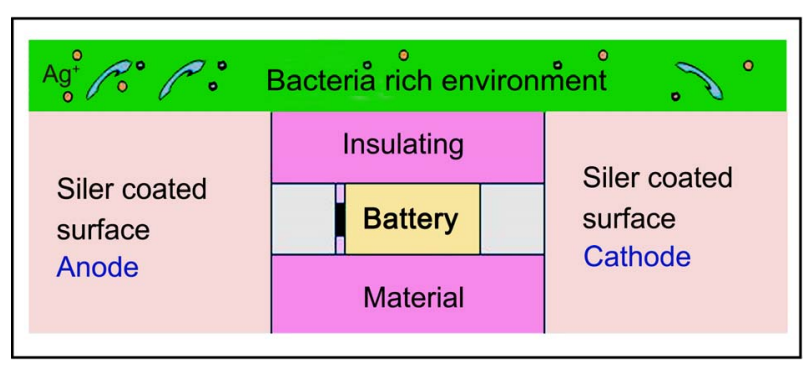

Figure 1. Proposed antimicrobial system concept.

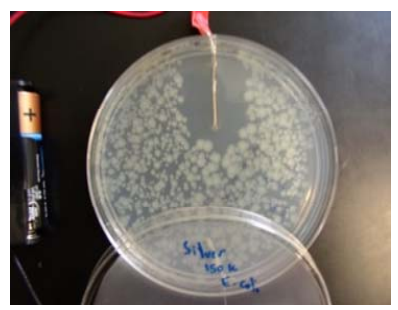

(a)

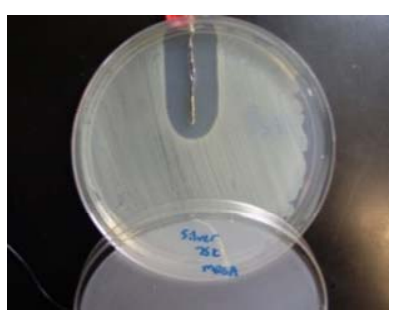

(c)

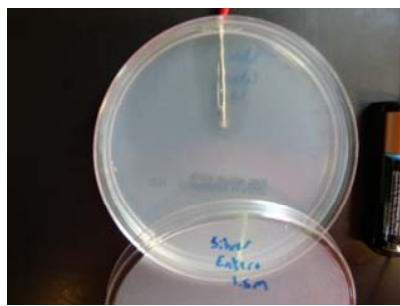

(b)

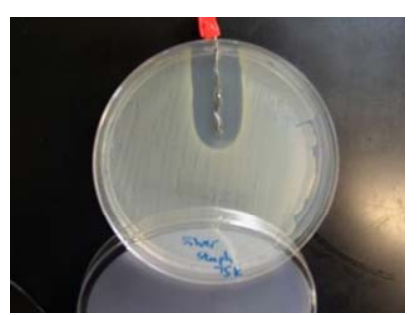

(d)
Figure 2. In vitro testing results of the proposed system on (a) Escherichia Coli; (b) Pseudomonas Aeruginosa; (c) Staphylococcus Aureus (d) MRSA. 


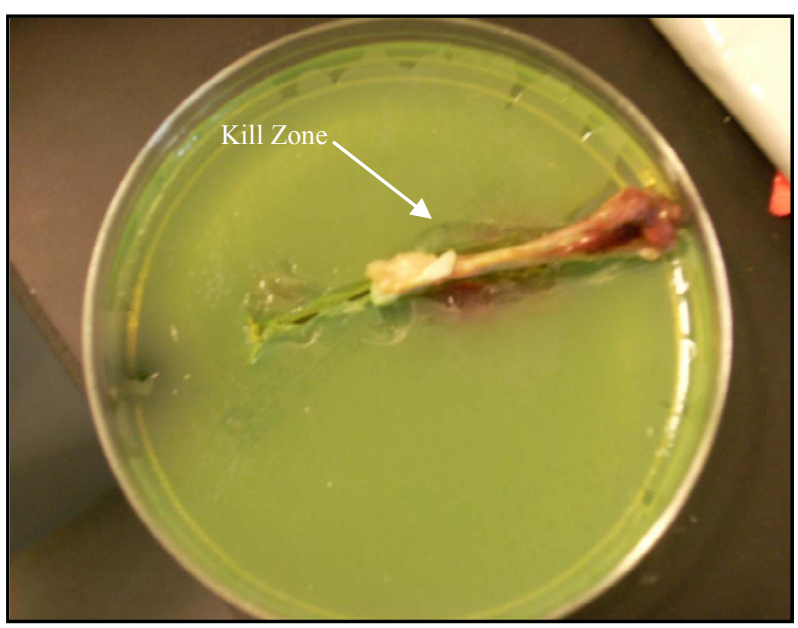

Figure 3. Simulated in vivo testing results of the proposed system on Pseudomonas Aeruginosa.

exposed to silver challenge the liver acts as a filter mechan ism, collecting over $90 \%$ of the absorbed silver and eliminating it in the feces via liver and biliary tract excretion $[16,17]$. As such the liver cells will experience the greatest levels of toxic elements borne by the blood. The cellular toxic levels of hepatocytes were determined to be both time and concentration dependant but could be appreciated at $30 \mu \mathrm{M}$ [18]. These results were confirmed by Rungby in 1990 when he proposed a range of 30-70 $\mu \mathrm{M}$ silver concentration as that needed to show appreciable cytotoxic effects within the hepatocytes [19]. Within all of our designs we will keep local ionic concentrateons at or below toxic concentrations by controlling the electrical circuitry associated with the implant device.

Two separate sets of animal testing studies were conducted to investigate the effectiveness of an in vivo electrically stimulated silver device that was inserted into the tibia's medullary cavity as a bactericidal agent. The protocol for generating osteomyelitis in the animals paralleled that described by Lucke et al. [20]. As described in this reference, rat tibias were inoculated with Staphylococcus aureus to try to create osteomyelitis. Surgical procedures were developed to install and retain the proposed bactericidal devices within the rat tibia.

The protocol to grow the inocula was adopted from Lucke et al. [21]. Staphylococcus aureus (ATCC number 29213), used as the pathogen, was grown overnight in $9 \mathrm{ml}$ tryptic soy broth (TSB) (caseinpepton-soybean flourpeptone-solution; Oxoid Ltd., Basingstoke, Hampshire, UK). From this culture, $100 \mu \mathrm{l}$ aliquots were transferred to sterile tubes containing $3 \mathrm{ml}$ of TSB. These tubes were then incubated for $3 \mathrm{~h}$ at $37^{\circ} \mathrm{C}$ to obtain log-phase growth. After incubation, the tubes were centrifuged for $10 \mathrm{~min}$ at $3000 \mathrm{rpm}$, the supernatant was decanted, and the remaining pellet was washed twice with phosphate- buffered saline (PBS). Under spectrophotometric control the bacterial sediment was added to PBS until a McFarland standard of six was obtained. Colony- forming units (CFU) per ml were confirmed by several plate counts with the use of a spiralplater (Spiral System Inc., Cincinnati, $\mathrm{OH}$ ). This procedure was repeated 20 times. Suspensions were split into portions and deep frozen at $-80^{\circ} \mathrm{C}$ until the day of surgery. To quantify a possible loss of viable bacteria following the freeze-thaw cycle the $\mathrm{CFU} / \mathrm{ml}$ were reconfirmed after each cycle of defrosting. In addition, each time a rat was inoculated, a $10 \mu \mathrm{sam}$ ple of inoculant was analyzed to determine its bacterial concentration. The concentration was adjusted to fit into the desired range $\left(10^{2}-10^{4} \mathrm{CFU} / \mathrm{ml}\right)$ using McFarland Standards and was confirmed by plate counts.

Surgeries in both sets of studies were performed at the Penn State Central Biology Labs. All through the testing studies, several different bactericidal devices designed were inserted into the tibial canal of 4-month-old Sprague Dawley rats through a proximal incision site and tibial cannulation. A similar surgical protocol was employed for all the hardware insertions with only slight device-specific modifications being made to the procedures as necessary. For each of the surgeries, one leg was shaved and scrubbed with betadine and alcohol prep. Each animal was placed on sterile drapes and their bodies were covered with sterile sheets; the prepped leg was separately draped in a sterile manner. A small incision $(\sim 5$ $\mathrm{mm}$ ) of skin and fascia at the proximal tibial metaphysis was created to provide access to the tibial periosteum. The medullary cavity of the proximal metaphysis was exposed using a $1 \mathrm{~mm}$ surgical drill, leaving the surrounding periosteum intact. A steel Kirschner wire (Ø $0.8 \mathrm{~mm})$ was inserted into the medullary cavity and pushed forward distally for smooth dilatation of the cavity for a length of approximately 8-10 mm distally, and removed. Staphylococcus aureus was injected into the medullary cavity to initiate osteomyelitis. Animals were closely monitored post-surgery and analgesics were administered initially on a regular schedule. No antibiotics were given to any of the animals since they would have masked the results of the test.

At the end of a two week incubation period, the animals were euthanized using carbon dioxide. The device was surgically removed and the anodic tip was used to inoculate a sheep blood agar plate. The tip of the device ( $5 \mathrm{~mm}$ ) was then cut and placed into $10 \mathrm{ml}$ of TSB. The blood agar plates and TSB were incubated for $24 \mathrm{~h}$ at $37^{\circ} \mathrm{C}$. Any CFUs found on the blood agar plate were counted and then analyzed to identify the bacterial species obtained from the tibia. The TSB was only visually examined for the presence of growth (cloudy: positive growth; clear: no growth). No further testing was perfor- 
med.

Initial bench top testing revealed that early prototype device designs performed well; however, biocompatibility within lab animals remained a question. Iterative testing of three device designs was performed to determine the one that was most compatible with the lab rats. Three separate, but similar, device-specific surgical procedures were used during this phase of the study.

\subsection{Overview of Surgical Procedure for Animal Testing Studies \#1}

The first set of animal testing studies was performed in 2007 in which three iterative designs of bactericidal devices and control devices were fabricated and inserted into the tibia medullary cavities of 21 living rats. Table 1 identifies the specifics of the iterative design changes. Sixteen of these animals survived the surgery and the two week incubation period following the surgery. Of the 16 rats, 5 were implanted with control devices (titanium wire). The inoculation process proved very successful as all 5 of the control animals without a bactericidal device developed Staphylococcus aureus based bone infections. Of the remaining 11 rats, only 9 animals had devices which remained in the tibia during the testing period. 3 of those 9 devices failed during the indwelling period leaving only 6 animals as prime targets for measuring efficacy of the device. Of these 6 possible cases, 3 of the rats with working devices were found to be staph free. All of the other animals with non-silver control devices or failed silver devices that were inoculated and euthanized cultured positive for Staphylococcus aureus. This preliminary study showed that the devices worked in at least half of the cases where they remained in the tibia and continued to be operational. The quantity of staphylococcus aureus present in the tibia was not quantified so it is possible that some of the devices judged ineffective may have significantly reduced the amount of bacteria present at the end of testing.

During this entire animal testing sequence, the surgi- cal procedures were continually refined making small iterative improvements for two purposes: 1) to keep foreign-borne microbes from impacting the testing and 2) to improve the device for animal comfort and performance. For surgeries performed on the first 6 rats, the staphylococcus $\left(\sim 10-20 \mu 1\right.$ containing $\left.10^{3} \mathrm{CFUs}\right)$ was injected via syringe into the medullary cavity prior to inserting the device. For the remainder of the surgeries, the wire tip of the device was dipped into the solution of staphylococcus aureus prior to inserting it into the tibia. This refinement was necessary because the medullary canal could not hold all of the fluid injected and the overflow ran into the soft tissue surrounding the incision site. The overflow of the injection fluid caused, in addition to the desired osteomyelitic infection, a soft tissue infection surrounding the incision site. Details of each of the procedures, blood reports and tibia X-ray reports were recorded. Ketmine/Xylazine $(100 \mathrm{mg} / \mathrm{kg})$ and IP Isoflurane $(10 \mathrm{mg} / \mathrm{kg})$ were used as anesthetics.

All three device designs used in this set of study and their surgical results are discussed below. One failure in this set of testing study was that the epoxy capsule used to enclose the device battery within the animal became infected in the majority of the animals.

\subsubsection{Antimicrobial Device \#1 —Design and Surgical Summary}

The first set of surgeries was performed on 6 rats. The first implant design had a stiff silver wire cathode $(\varnothing 0.8$ $\mathrm{mm}, \sim 30 \mathrm{~mm}$ length) and a stiff silver wire anode (Ø $0.8 \mathrm{~mm}, \sim 40 \mathrm{~mm}$ length and then cut to fit the tibia) that was inserted into the tibial canal. The anodic wire was connected to the $1.5 \mathrm{~V}$ battery via a 35 gage insulated copper wire. The conductive path for the two wires was the highly resistive soft rat tissue between the anode and cathode. The distance between the two wires (anode and cathode) was $\sim 70-90 \mathrm{~mm}$ when implanted, and the total length of the flexible wire was as much as $120 \mathrm{~mm}$. A small epoxy cap was used to prevent the battery and wires from eroding through the external skin layers. The

Table 1. Device design summary for 2007 animal testing.

\begin{tabular}{ccccccc}
\hline $\begin{array}{c}\text { Section of } \\
\text { Text }\end{array}$ & $\begin{array}{c}\text { Device } \\
\text { number }\end{array}$ & $\begin{array}{c}\text { Figure } \\
\text { number }\end{array}$ & Anode configuration & Cathode configuration & Length of device & $\begin{array}{c}\text { Battery } \\
\text { encasement }\end{array}$ \\
\hline 4.11 & 1 & 4 & $\begin{array}{c}\text { Stiff silver wire - connected to battery } \\
\text { by flexible 35 gauge copper wire }\end{array}$ & Stiff silver wire & $100-120 \mathrm{~mm}$ & epoxy \\
\hline 4.12 & 2 & 5 & $\begin{array}{l}\text { Stiff silver wire - connected to battery } \\
\text { by flexible 35 gauge copper wire }\end{array}$ & Flexible copper wire & $80-100 \mathrm{~mm}$ & epoxy \\
\hline 4.13 & 3 & 6 & $\begin{array}{l}\text { Stiff silver wire - connected to battery } \\
\text { by flexible 35 gauge copper wire }\end{array}$ & $\begin{array}{c}\text { Silver painted wire, } \\
\text { wrapped around insulated } \\
\text { portion of anode wire }\end{array}$ & $80-90 \mathrm{~mm}$ & epoxy \\
\hline
\end{tabular}


device design for these surgeries is shown in Figure 4. The anodic tip of the device was inserted into the tibial canal; the conductive wire and battery pack was stored under the dermatological layer of the rat's flank. This design was tested at two different levels of current - high amperage (without resistor) and low amperage (with resistor), and with a similar control device in which the battery was removed and the silver wire was replaced with a titanium wire (2 rats).

Thus, six rats were fit with implants during the first set of surgical trials; two devices with no resistor, two devices with $1 \mathrm{M}-\Omega$ resistor and two devices with titanium wire (control). All 6 rats survived both the surgery and the two week incubation period post-surgery. After the two week period, all 6 rats were euthanized and studied to determine if the devices had eliminated the bacteria (especially the staphylococcus aureus). All of the devices were checked for continuity and voltage after removal. Three of the four devices were deemed to be functional. 2 of these 3 rats with functional devices did not show any staph growth.

\subsubsection{Antimicrobial Device \#2-Design and Surgical Summary}

The second set of surgeries was performed on 9 rats. In this set of surgeries, the cathode of the implanted device was modified by replacing the stiff sliver wire with a flexible copper wire. The additional flexibility was desired for rat comfort as the original design (stiff wire) dug into the rat's muscle tissue, causing a point of irritation. The total length of the implant was reduced to $\sim 80-100 \mathrm{~mm}$ in order to facilitate ease of insertion. The inoculation during the second surgery set was performed by dipping the wire into the staphylococcus aureus solution to induce a staph infection within the medullary cavity. The device used for the second set of surgeries is shown in Figure 5.

Nine rats were surgically implanted with the following: three devices with no resistor (high amperage), three with a $1 \mathrm{M}-\Omega$ resistor (low amperage) and three with a titanium control. In this group, 2 of 9 rats died. The remaining 7 rats survived the observation period and were euthanized after two weeks. A few of the devices had their cathode tipping out of the rat skin, thus breaking the continuity while in vivo. Devices were checked for continuity after the animals were euthanized and only three appeared functional. Unfortunately, the design changes created a device that could "float" within the animals, allowing the devices to migrate out of the medullary cavity during the post-surgical phase of the study. Due to migration, none of the devices remained in the tibial canal when the postmortems were performed. Drawbacks of this design included breakage of the copper wire and poking of the cathode through the rat skin. Resistive soft tissue between the anode-cathode separation was also a problem.

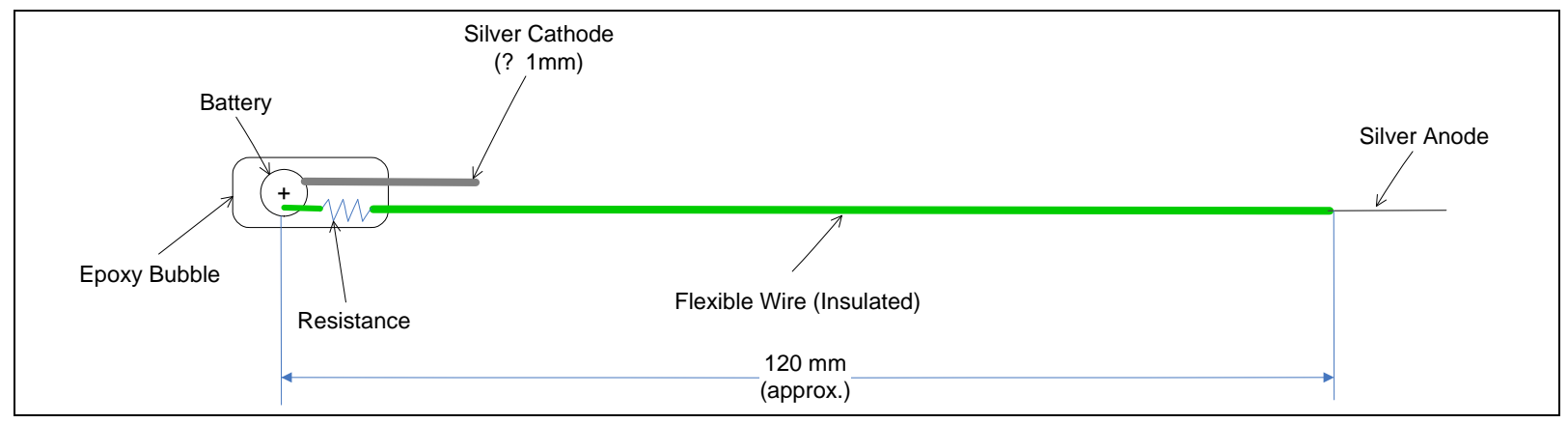

Figure 4. Antimicrobial device \#1.

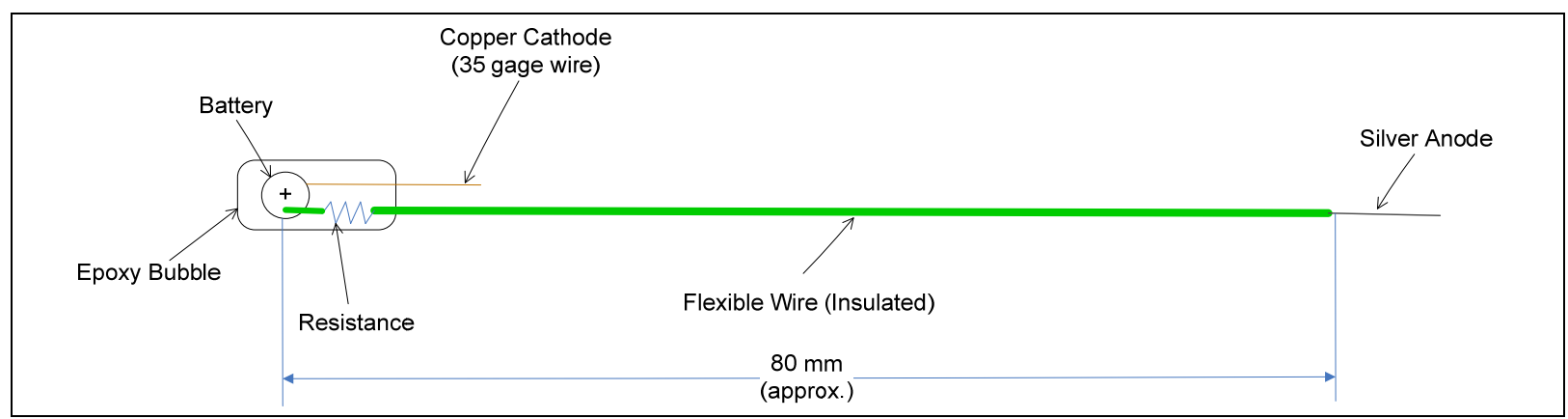

Figure 5. Antimicrobial device \#2. 


\subsubsection{Antimicrobial Device \#3 - Design and Surgical Summary}

In order to alleviate the migration of the device within the test animal, a third device design was created. The anode and cathode were integrated on the same wire and were separated by a small insulation strip. In this configuration, electrons are drawn from the silver paint (cathode) to the anode over the insulation through the conducting media. This design was accompanied by a change in electrode polarities resulting in two types of devices anodic and cathodic. The length of the flexible wire was limited to $\sim 80-90 \mathrm{~mm}$. Similar to the previous sets of surgeries, bacterial inoculation was created by dipping devices into the staphylococcus aureus solution. The third device design is shown in Figure 6.

Within this phase of the study, 6 rats were surgically implanted using two implants of each kind: three with high amperage anodic devices, two with low amperage anodic devices and one with a high amperage cathodic device. In this group, 3 of the 6 rats died post surgery. The remaining 3 rats survived for the full observation period and were euthanized after one week. All the devices were checked for continuity and voltage after removal and all were functional.

Several modifications to the implant device and surgical procedures were made. The device changes appeared to have corrected most of the early drawbacks. The surgical procedure was modified to include a stitch to hold the device in place. The result was that none of the devices floated within the rat's body. Only 1 of the 3 rats which survived was staph free. This could have been due to the limited separation between the electrodes. This device appeared to be working as evident by the lack of puss on gross examination during the device removal; however a quantitative CFU count was not performed.

A few potential device design concerns remained for this device. It was possible that the amount of fluids surrounding the implant was insufficient to facilitate proper movement of the antimicrobial $\mathrm{Ag}^{+}$ions. Another possibility was that the flow of antimicrobial $\mathrm{Ag}^{+}$ions was localized since ions take the path of least resistance and in this case the path of least resistance translated into the path of least distance over the insulation (about $5 \mathrm{~mm}$ ).

\subsection{Overview of Surgical Procedure for Animal Testing Studies \#2}

The second set of animal testing surgeries was performed in 2008. A total of 16 surgeries were performed with 4 rats used as controls (titanium wire/silver wire without current) and 12 rats implanted with three iterations of antimicrobial designs. Table 2 identifies the specifics of the iterative design changes. Thirteen of these animals survived the surgery and an incubation period and could be harvested for pathology. Three of these thirteen animals ( 2 with antimicrobial devices and 1 with control) were harvested within the one week incubation period following the surgery due to declining health. Thus, 3 animals with control and 10 animals with the antimicrobial devices were left as prime targets for measuring the efficacy of the devices. All control rats tested positive for Staphylococcus aureus growth. Where the devices failed, all of the animals also tested positive for Staphylococcus aureus. Of the 10 rats that had antimicrobial devices implanted and survived the surgery and incubation period, 4 rats were clear of Staphylococcus aureus. The Staph growth was not quantified in this study.

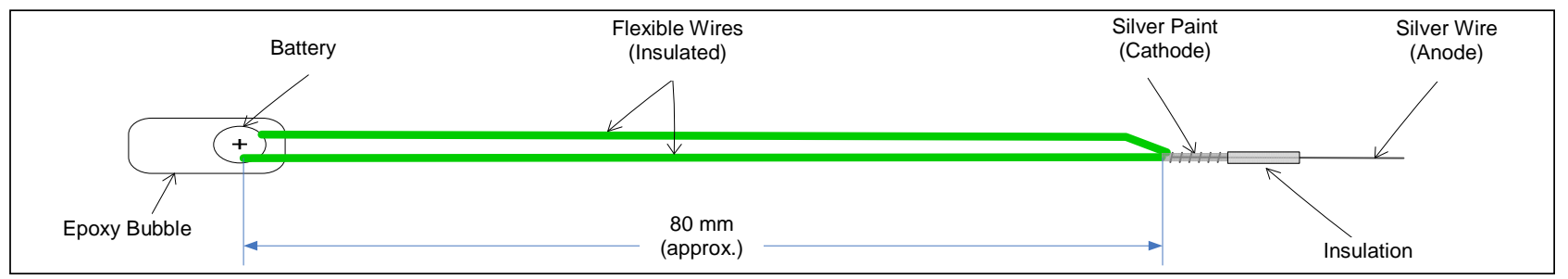

Figure 6. Antimicrobial device \#3.

Table 2. Device design summary for 2008 animal testing.

\begin{tabular}{cccllcc}
\hline $\begin{array}{c}\text { Section of } \\
\text { Text }\end{array}$ & $\begin{array}{c}\text { Device } \\
\text { number }\end{array}$ & $\begin{array}{c}\text { Figure } \\
\text { number }\end{array}$ & Anode configuration & \multicolumn{1}{c}{ Cathode configuration } & $\begin{array}{c}\text { Length of } \\
\text { device }\end{array}$ & $\begin{array}{c}\text { Battery } \\
\text { encasement }\end{array}$ \\
\hline 4.21 & 4 & 7 & $\begin{array}{l}\text { Stripped end of Teflon } \\
\text { insulated silver wire }\end{array}$ & $\begin{array}{l}\text { Stripped end of Teflon insulated silver wire, } \\
\text { wrapped around insulated portion of anode wire }\end{array}$ & $60 \mathrm{~mm}$ & epoxy \\
\hline 4.22 & 5 & 8 & $\begin{array}{l}\text { Stiff silver wire connected } \\
\text { directly to battery }\end{array}$ & $\begin{array}{l}\text { Stripped end of copper wire, wrapped around } \\
\text { section of anode, insulated via heat shrink wrap }\end{array}$ & $60 \mathrm{~mm}$ & Silicone \\
4.23 & 6 & 9 & $\begin{array}{l}\text { Stiff silver wire connected } \\
\text { directly to battery }\end{array}$ & $\begin{array}{l}\text { Stripped end of Teflon insulated silver wire, } \\
\text { wrapped around section of anode, insulated via } \\
\text { heat shrink wrap }\end{array}$ & $45 \mathrm{~mm}$ & Silicone \\
\hline
\end{tabular}


Refinements in this set of surgeries were focused on keeping foreign-borne microbes from impacting the testing, and improving the device for animal comfort and performance. A surgical protocol similar to the one used in the first set of surgeries was followed. Two primary modifications in the surgeries were made: 1) the wire device was inserted in the primal portion of the tibia so that a longer portion of the device could be inserted, and 2) the animals were placed in a plastic surgical sack so that airborne bacteria could be contained. In this set of surgeries, the medullary cavity of the primal metaphysis was exposed using a 16 gage needle to produce a hole. 5 $\mu \mathrm{l}$ of Staphylococcus aureus $\left(10^{4} \mathrm{CFU} / \mathrm{ml}\right)$ was injected via micro-syringe into the medullary cavity prior to inserting the device. The micro-syringe eliminated any overflow of the bacteria. Similar to the first set of surgeries, all animals were closely monitored post-surgery and analgesics were administered initially on a regular schedule. No antibiotics were given to any of the animals to eliminate masking of test results. The epoxy capsule used to enclose the device battery within the animal in our early testing was replaced by a silicone shell to minimize discomfort to the animals.

\subsubsection{Antimicrobial Device \#4-Design and Surgical Summary}

The fourth implant design used during the first set of surgeries in the second study set was a modification of antimicrobial device \#3 used during the first set of study. In this design, the anode and cathode were integrated on the same wire. Unlike device \#3 where the anode was a silver wire soldered to 35 gage copper wire, the anode in this design was the stripped end of Teflon coated silver wire directly connected to the positive terminal of a 1.5 $\mathrm{V}$ battery. Similarly, the silver painted cathode of device \#3 was replaced by the stripped end of another Teflon coated silver wire directly connected to the negative battery terminal. The electrodes were separated by Teflon insulation on the anodic wire. The electrons were drawn from the silver anode to the silver cathode over the insu- lation by the conducting media. A small silicone cap was used to encase the battery and its soldered connections. Length of the device was limited to $60 \mathrm{~mm}$. The design was used with two combinations of currents - high amperage (no resistor) and low amperage (100 $\mathrm{K} \Omega$ resistor). Figure 7 shows schematic drawing of the high amperage device design.

Four rats were implanted with this device and all survived the surgery and incubation period. One of the 4 rats implanted with this device tested staph free. This result could be attributed to the quality of the fabricated device as all devices seemed to separate at the coiled portion. Although this design was more robust in construction than the previous ones, a more effective release of ions was desired. This could be achieved by replacing one of the coated electrode wires with a bare one. Commercial silicone used to encase the battery was not bio-compatible and caused irritation to the rats.

\subsubsection{Antimicrobial Device \#5-Design and Surgical Summary}

Since anodic device designs were being used, the Teflon coated silver anode was replaced by a bare silver wire. Thirty-five gage copper wire was used as the cathode. Insulated shrink tubing was fitted onto the bare silver anode to separate the electrodes. Commercial silicone was replaced with medical grade sterile silicone for the battery encasing. Length of the device was limited to 60 $\mathrm{mm}$. These devices were only used with high amperage (no resistor). The device design is shown in Figure 8.

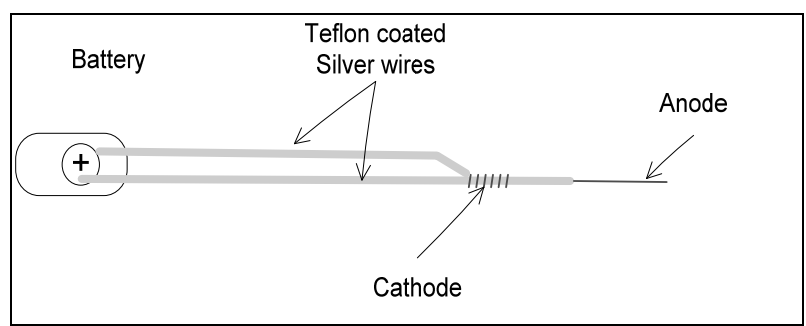

Figure 7. Antimicrobial device \#4.

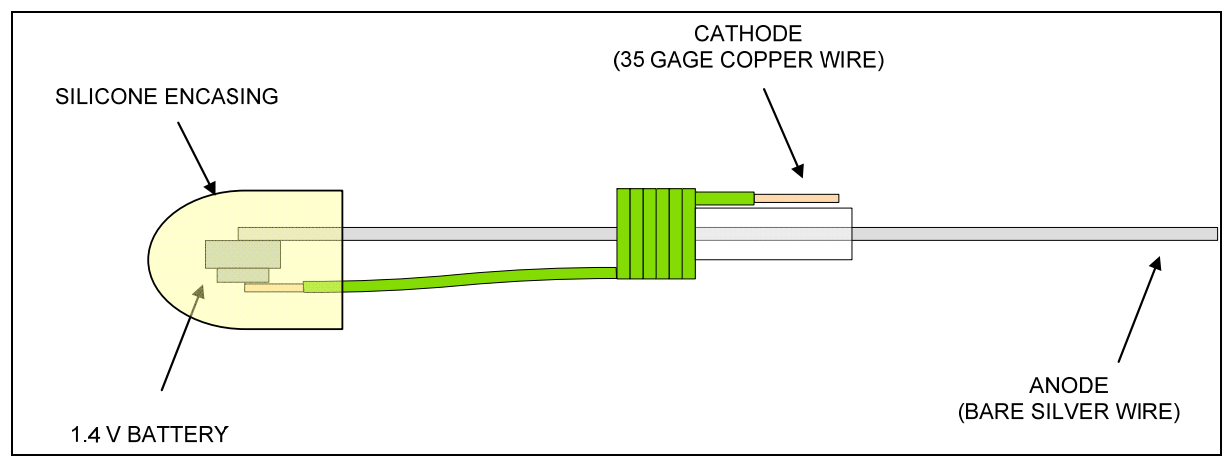

Figure 8. Antimicrobial device \#5. 
Of the 4 rats implanted with antimicrobial devices, 1 died before pathology was performed. One of the remaining 3 with working antimicrobial devices tested Staph free. In analyzing the device used in these surgeries, we concluded that the 35 gage copper wire should be replaced with Teflon coated silver wire as the cathode as the copper wire seemed to unravel and oxidize. The silicone encasing should be reduced in size by about $20 \%$. The length of the device should be smaller for ease of insertion.

\subsubsection{Antimicrobial Device \#6 - Design and Surgical Summary}

This device was slightly modified from the previous version. Bare silver wire continued to be used as the anode. Teflon coated silver wire replaced 35 gage copper wire as the cathode. In addition to the shrink tubing used as insulation between the anode and cathode, another piece of the tubing was introduced to hold the anodic and cathodic wires together. The size of medical grade silicone encapsulation was reduced. Length of the device was reduced to $45 \mathrm{~mm}$. These devices were also used with high amperage (no resistor). The schematic design of the device is shown in Figure 9.

Four rats were implanted with this device out of which 3 survived through the post surgery incubation period.
Two of the remaining 3 rats were found to be Staph free. Of the 10 rats that had antimicrobial implant devices and survived through the incubation period post surgery in animal testing studies \#2, the rats implanted with these devices looked the healthiest. This design eliminated all drawbacks of the previous versions and seemed very robust.

\section{STATISTICAL ANALYSIS}

Statistical testing was conducted in order to determine any significant statistical difference between the devices used in surgeries. Because several of the animals died prior to harvest and 3 of the devices did not function after they were implanted, unequal test samples resulted for all categories. This made performing a paired test impossible so an F-Test was conducted to determine significance. The first test conducted compared all effective control devices to all effective antimicrobial devices. The term effective here means that the devices remained in the tibia post-surgery and were extracted from the live rats after an incubation period. These results are summarized in Table 3. Using a Fischer's F-Test at 85\% confidence level, we can conclude that there is a statistically signify- cant difference between antimicrobial and control devices.

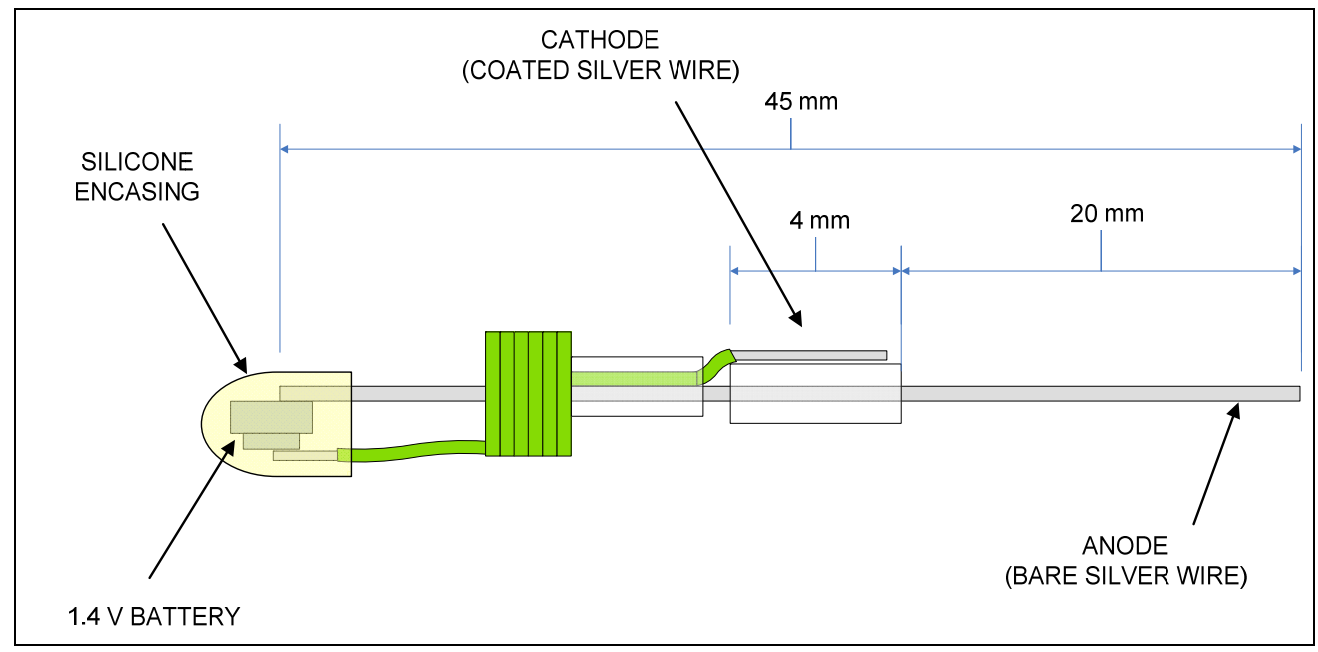

Figure 9. Antimicrobial device \#6.

Table 3. Summary of all devices for all surgeries (total)

\begin{tabular}{cccc}
\hline & Staph aureus free & Staph aureus & Total \\
\hline Antimicrobial device & 7 & 12 & 19 \\
Control Device & 0 & 7 & 7 \\
Total & 7 & 19 & 26 \\
\hline
\end{tabular}


During animal testing studies \#1, antimicrobial devices were found to be electrically disconnected proximal to the tibial cavity insertion point of 2 rats, rendering the devices electrically ineffective. If these 2 devices are considered as control instead of antimicrobial devices, the F-test concludes that there is a statistically significant difference between antimicrobial devices and control devices at $95 \%$ confidence level. In addition, each device design was compared with others to determine any statistical significance. Strictly statistically speaking, only antimicrobial device \#1 and antimicrobial device \#6 showed significant difference from the controls among all six design iterations. Although 2 out of 3 animals implanted with working antimicrobial device \#1 tested staph free, the device tip often penetrated the soft tissue and caused irritation to the animals. On the other hand, antimicrobial device \#6 also resulted in 2 out of 3 animals being staph free but eliminated the problem associated with damaging the soft tissue.

\section{DISCUSSION AND CONCLUSIONS}

An early conjecture in this research was that a bactericidal environment would be created if we could get bacteria to conduct $\mathrm{Ag}^{+}$. The hope was to create this environment within a bone because getting traditional antibiotics to penetrate the bone while the antibiotics are still viable can be very difficult. A basic design concept was developed and had to go through several iterations governed by rat comfort after implant as well as bactericidal performance. Interestingly, the first and the last device design iterations showed the greatest efficacy.

Based on the surgeries, pathology results and statistical analysis, the rat osteomyelitic model described in literature is validated, since all animals without an antimicrobial device were infected. More importantly, the results show that properly configured electrically stimulated silver is an effective bactericidal agent for indwelling devices. Of all the surgeries performed using the bactericidal devices, there is a statistically significant difference between using no device and an antimicrobial device. As the antimicrobial devices and surgical procedures were refined throughout the study, the effectiveness of the devices was found to be improved. In the last set of surgeries, $67 \%$ of the harvested animals were free of Staphylococcus aureus even after they were inoculated with the bacteria and given no antibiotics. The bactericidal device as configured has a definite ability to reduce/ eliminate bacterial infection. Using such a bactericidal device in conjunction with a standard treatment of antibiotics should have a profound effect on the number of residual hardware associated bacterial infections.

\section{ACKNOWLEDGEMENTS}

The technology and designs tested within this study are protected under U. S. Patent as owned by ArgentumCidalElectrics, Inc.. It is only with their support that device modifications and manufacturing could be properly completed and controlled.

\section{REFERENCES}

[1] Iorio, R., Robb, W.J., Healy, W.L., Berry, D.J., Hozack, W.J., Kyle, R.F., Lewallen, D.G., Trousdale, R.T., Jiranek, W.A., Stamos, V.P. and Parsley, B.S. (2008) Orthopaedic surgeon workforce and volume assessment for total hip and knee replacement in the united states: Preparing for an epidemic. The Journal of Bone and Joint Surgery, 90(7), 1598-1605.

[2] Kurtz, S., Ong, K., Lau, E., Mowat F. and Halpern, M. (2007) Projections of primary and revision hip and knee arthroplasty in the United States from 2005 to 2030. The Journal of Bone and Joint Surgery, 89(4), 780-785.

[3] Cumming, D. and Parker, M.J. (2007) Urinary catheterisation and deep wound infection after hip fracture surgery. International Orthopaedics, 31(4), 483-485.

[4] Fitzgerald Jr.R.H. (1992) Total hip arthroplasty sepsis: Prevention and diagnosis. Orthopedic Clinics of North America, 23(2), 259-264.

[5] Furst, A. and Schlauder, M.C. (1978) Inactivity of two noble metals as carcinogens. The Journal of Environmental Pathology, Toxicology and Oncology, 1(1), 5157.

[6] Solberg, B.D., Gutow, A.P. and Baumgaertner, M.R. (1999) Efficacy of gentamycin impregnated resorbable hydroxyapatite cement in treating osteomyelitis in a rat model. Journal of Orthopaedic and Trauma, 13(2), 102106.

[7] Furno, F., Morley, K.S., Wong, B., Sharp, B.L., Arnold, P.L., Howdle, S.M., Bayston, R., Brown, P.D., Winship P.D. and Reid, H.J. (2004) Silver nanoparticles and polymeric medical devices: A new approach to prevention of infection? The Journal of Antimicrobial Chemotherapy, 54(6), 1019-1024.

[8] Klasen, H.J. (2000) Historical review of the use of silver in the treatment of burns Part 1: Early uses. Burns, 26(2), 117-130.

[9] Klasen, H.J. (2000) Historical review of the use of silver in the treatment of burns Part 2: Renewed interest for silver. Burns, 26(2), 131-138.

[10] Samuel, U. and Guggenbichler, J.P. (2004) Prevention of catheter-related infections: The potential of a new nanosilver impregnated catheter. The International Journal of Antimicrobial Agents, 23(Suppl 1), 75-78.

[11] Langreth, R. and Herper, M. (2006) Germ Warfare. Forbes http://www.forbes.com/forbes/2006/0619/060.html.

[12] Melaiye, A. and Youngs, W.J. (2005) Silver and its application as an antimicrobial agent. Expert Opinion on Therapeutic Patens, 15(2), 125-130.

[13] Price, W.R. and Wood, M. (1996) Silver nitrate burn dressing: Treatment of seventy burned persons. American Journal of Surger , 112(5), 674-680.

[14] Weber, D.J. and Rutala, W.A. (2001) Use of metals as 
microbicides in preventing infections in healthcare. In: Block SS. Disinfection, sterilization, and preservation, Lippincott Williams \& Wilkins, Philadelphia, PA, 415-430.

[15] Slawson, R.M., Van Dyke, M.L., Lee, H. and Trevors, T. J. (1992) Germanium and silver resistance, accumulation and toxicity in microorganisms. Plasmid, 27(1), 72-79.

[16] Alexander, J. and Aaseth, J. (1981) Hepatobillary transport and organ distribution of silver in the rat as influenced by selenite. Toxicology, 21(3), 179-186

[17] Fowler, B.A. and Nordberg, G.F. (2007) Silver. In: Friberg, G.F. Nordberg and V. Vouk (Eds.), Handbook on the toxicology of Metals, 2nd Edition, Elsevier, Amsterdam, 521-531.

[18] Baldi, C., Minoia, C., Di Nucci, A., Capodagio, E and Manso, L. (1988) Effects of silver in isolated rat hepato- cytes. Toxicology Letters, 41(3), 261-268

[19] Rungby, J. (1990) An experimental study of silver on the nervous system and on aspects of its general cellular toxicity. Danish Medical Bulletin, 37(5), 442-449

[20] Lucke, M., Wildemann, B., Sadoni, S., Surke, C., Schiller, R., Stemberger, A., Raschke, M., Haas, N.P. and Schmidmaier, G. (2005) Systematic versus local application of gentamicin in prophylaxis of implant-related osteomyelitis in a rat model. Bone, 36(5), 770-778.

[21] Lucke, M., Schmidmaier, G., Sadoni, S., Wildemann, B., Schiller, R., Stemberger, A., Haas, N.P. and Raschke, M. (2003) A new model of implant related osteomyelitis in rats. Journal of Biomedical Materials Research, 67B(1), 593-602. 